\title{
Temperature dependence of photoluminescence in non-crystalline silicon
}

\author{
Ali Serpengüzel, ${ }^{*}$ Temel Bilici, ${ }^{\dagger}$ Ibrahim Inanç, ${ }^{\ddagger}$ and Adnan Kurt \\ Koç University, Microphotonics Research Laboratory, Department of Physics, \\ Rumeli Feneri Yolu, Sariyer, Istanbul 34450 Turkey \\ Jim Carey and Eric Mazur \\ Harvard University, Department of Physics and Division of Engineering and Applied Sciences, \\ 29 Oxford Street, Cambridge, Massachusetts 02138 USA
}

\begin{abstract}
Crystalline silicon being ubiquitous throughout the microelectronics industry has an indirect bandgap, and therefore is incapable of light emission. However, strong room temperature visible and near-IR luminescence from non-crystalline silicon, e.g., amorphous silicon, porous silicon, and black silicon, has been observed. These silicon based materials are morphologically similar to each other, and have similar luminescence properties. We have studied the temperature dependence of the photoluminescence from these non-crystalline silicons to fully characterize and optimize these materials in the pursuit of obtaining novel optoelectronic devices.
\end{abstract}

Keywords: amorphous silicon, black silicon, non-crystalline silicon, porous silicon, plasma enhanced chemical vapor deposition, photoluminescence..

\section{INTRODUCTION}

Interest in silicon ( $\mathrm{Si}$ ) as a material for optoelectronics has increased recently. With modern process techniques, it will be possible to integrate lasers, photodetectors, and waveguides into optoelectronic silicon motherboards to route and modulate optical signals within such silicon motherboards. Integrated silicon optoelectronics is a rapidly developing field ${ }^{1}$. Discrete and integrated devices such as photodetectors, modulators, light emitters, resonant cavity enhanced (RCE) photodetectors, waveguides, photonic bandgap filters, optical amplifiers, optical interconnects, and optoelectronic integrated circuits are already being realized. However, most of these devices, with the exception of light emitters, are fabricated using crystalline silicon. Light emission requires the use of amorphous silicon, since crystalline silicon can not emit light due to its indirect bandgap. With modern process techniques, it will be possible to integrate lasers, photodetectors, and waveguides into $\mathrm{Si}$ motherboards ${ }^{2,3}$ for wavelength division multiplexing (WDM) applications ${ }^{4}$.

\section{EXPERIMENTAL PHOTOLUMINESCENCE SETUP}

The room temperature PL setup consists of a pump laser, a spectrometer, a lock-in amplifier, and a digital oscilloscope. The collected PL signal is imaged to the entrance slit of the spectrometer, whose output is fed to a sensitive photomultiplier tube (PMT). A lock-in-amplifier is providing the necessary electronic gain to the PMT output gain in phase with the laser pulse. The digital oscilloscope is used for monitoring and optimizing the PMT signal. All of the

* aserpenguzel@ku.edu.tr; phone: + 90 (212) 338-1312; fax: + 90 (212) 338-1547, http://home.ku.edu.tr/ aserpenguzel, http://microphotonics.ku.edu.tr, Koç University, Microphotonics Research Laboratory, Department of Physics, Rumeli Feneri Yolu, Sariyer, Istanbul 34450 Turkey. Ibrahim Inanç and Adnan Kurt are with Koç University, Microphotonics Research Laboratory, Department of Physics, Rumeli Feneri Yolu, Sariyer, Istanbul 34450 Turkey. $\dagger$ Temel Bilici is currently at Boston University, Department of Electrical and Computer Engineering, 8 St. Mary's Street, Boston, Massachusetts 02215 USA. \$ Ibrahim Inanç is also with the Bogaziçi University, Physics Department, Bebek, Istanbul 34342 Turkey. Jim Carey and Eric Mazur are with Harvard University, Department of Physics, Cambridge, Massachusetts 02138 USA. 
measurement and test devices are computer controlled and the data is acquired digitally. For the room temperature measurements at $300 \mathrm{~K}$ the samples are attached to a holder. For low temperature measurements the samples are placed in a closed cycle cryostat system. The closed cycle cryostat system is used to control the sample temperature from $10 \mathrm{~K}$ to $300 \mathrm{~K}$.

\section{AMORPHOUS SILICON}

In the optoelectronics and microelectronics industry silicon is the most widely used semiconductor, not only in its crystalline, but also in its amorphous form. Being a direct band-gap material, ${ }^{5}$ unlike crystalline silicon, ${ }^{6}$ amorphous silicon is unmatched as a photoreceptor for laser printing, for switching elements in large area liquid crystal displays, for large photovoltaic panels, and any other application that calls for a high quality semiconductor that can be processed on large areas or on curved or flexible substrates. It is generally agreed that the terms amorphous solid, non-crystalline solid, disordered solid, glass, or liquid have no precise structural meaning beyond the description that, the structure is not crystalline on any significant scale. The principal structural order present is imposed by the approximately constant separation of nearest-neighbor atoms or molecules. Until the early 1970's, amorphous silicon prepared by evaporation or sputtering was not considered as one of the valuable semiconductor materials, because of high density of electronic states in the band gap related to a large density of structural defects. ${ }^{7}$ The discovery of an amorphous silicon material prepared by the glow discharge deposition of silane, which can be doped and whose conductivity can be changed by ten orders of magnitude marked a turning point and opened a new research area. Most interest focused on hydrogenated amorphous silicon and its alloys, since hydrogen by removing dangling bonds eliminates non-radiative recombination centers, that are responsible for reduced luminescence efficiency (and reduced photovoltaic efficiency) and allows doping. ${ }^{8}$ Hydrogenated amorphous silicon $(\mathrm{a}-\mathrm{Si}: \mathrm{H})$ is already an established material in semiconductor technology ${ }^{9}$. The major application of a-Si:H is photovoltaics, color detectors, and active matrix displays. The primary attribute of the technology is its large area capability, which is unavailable with other technologies. Another advantage of the hydrogenated amorphous silicon is that, it can be deposited by plasma enhanced chemical vapor deposition (PECVD) onto almost any substrate at temperatures below $500 \mathrm{~K}$, which makes it compatible with the microelectronic technology. This property justifies the interest in a-Si:H as a potential optoelectronic material. Planar waveguides are already being realized from a-Si:H ${ }^{10}$. The advantage of a-Si:H, as well as porous silicon (п-Si), is that, they attract interest as a potential optical gain medium, because of their room temperature visible electroluminescence (EL) and photoluminescence (PL). Recently, we have observed visible PL from a-Si:H, as well as its oxides $\left(\mathrm{a}-\mathrm{SiO} \mathrm{x}_{\mathrm{x}}: \mathrm{H}\right)$ and nitrides $\left(\mathrm{a}-\mathrm{SiN}_{\mathrm{x}}: \mathrm{H}\right)$ grown by low temperature PECVD. ${ }^{11}$ While the exact mechanism of the occurrence of the PL in bulk a-SiN ${ }_{x}: H$ is still under discussion, the quantum confinement model is a widely accepted ${ }^{12}$ In the quantum confinement model the material consist of small a-Si clusters in a matrix of a-SiN ${ }_{x}: \mathrm{H}$. The regions with $\mathrm{Si}-\mathrm{H}$ and $\mathrm{Si}-\mathrm{N}$, having larger energy gaps due to strong $\mathrm{Si}-\mathrm{H}$ and $\mathrm{Si}-\mathrm{N}$ bonds, isolate these a-Si clusters, and form barrier regions around them. The PL originates from the a-Si clusters. A-SiN $: \mathrm{H}$ can be grown both with and without ammonia $\left(\mathrm{NH}_{3}\right)$. The samples grown without $\mathrm{NH}_{3}$ are referred to as the Si rich samples. The luminescence of these samples is in the rednear-infrared part of the optical spectrum. The samples grown with $\mathrm{NH}_{3}$ and annealed at $800^{\circ} \mathrm{C}$ are referred to as the nitrogen rich samples. The luminescence of these samples is in the blue-green part of the optical spectrum ${ }^{13}$.

\subsection{Temperature dependence of the amorphous silicon photoluminescence}

Figure 3.1 shows the PL spectra of the $\mathrm{a}-\mathrm{SiN}_{\mathrm{x}}: \mathrm{H}$ measured in the $550-900 \mathrm{~nm}$ wavelength and in the $12-298 \mathrm{~K}$ temperature range at a constant excitation laser intensity of $0.1 \mathrm{~W} \mathrm{~cm}^{-2}$. A broad PL band centered at $710 \mathrm{~nm}\left(E_{p}=\right.$ $1.746 \mathrm{eV}$ ) at $12 \mathrm{~K}$ is observed. The PL spectra have approximately a Gaussian lineshape modulated slightly by FabryPerot resonances. These resonances are due to the Fresnel reflections from the a-SiN $\mathrm{x}_{\mathrm{x}} \mathrm{H}$ film surfaces. The PL intensity decreases with increasing temperature. This feature is typical of the PL, which is due to donor-acceptor pair transitions observed in semiconductors. The variation of the PL peak with respect to temperature is plotted in the figure 3.2. In the 12 - $170 \mathrm{~K}$ range, the PL intensity decreases slowly. Above $170 \mathrm{~K}$, however, the PL intensity decreases at a larger rate due to a thermal quenching process. The activation energy $\triangle \mathrm{E}$ for this thermal quenching process can be derived in the 170 - $298 \mathrm{~K}$ temperature range using a nonlinear least squares fit to the following equation, $\mathrm{I}=\mathrm{I}_{0} \exp \left(\Delta \mathrm{E} / \mathrm{k}_{\mathrm{B}} \mathrm{T}\right)$, where I is the PL intensity, $I_{o}$ a proportionality constant, and $k_{B}$ the Boltzmann's constant. The semilog plot of the emission band intensity as a function of the reciprocal temperature gives a straight line in the $170-298 \mathrm{~K}$ region. An activation energy of $E_{a}=0.027 \mathrm{eV}$ for the emission band is derived from the slope of the straight line fit. This activation energy is associated with a shallow level located at $0.027 \mathrm{eV}$ from the band. This shallow level in undoped a-SiN $\mathrm{N}_{\mathrm{x}}: \mathrm{H}$ may be associated with the presence of defects and unintentional impurities. 


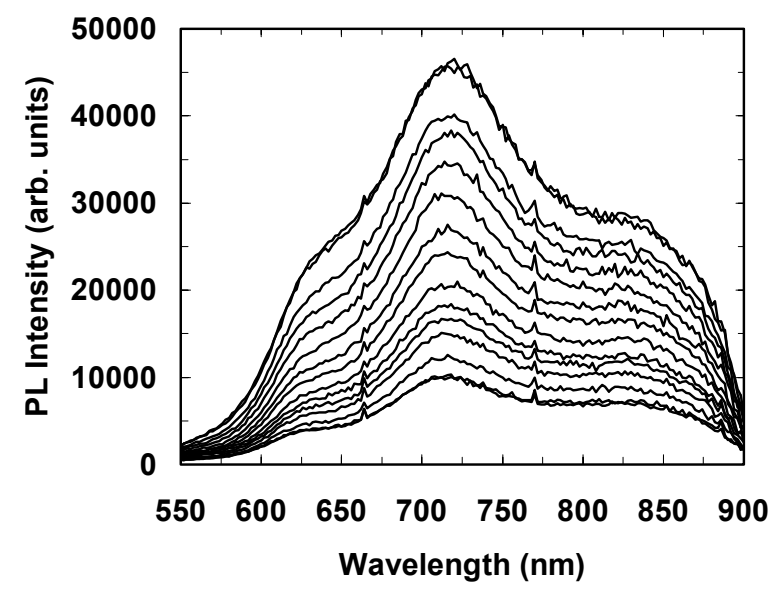

Figure 3.1. PL spectra of a-SiNx:H in the $12-298 \mathrm{~K}$ temperature range.

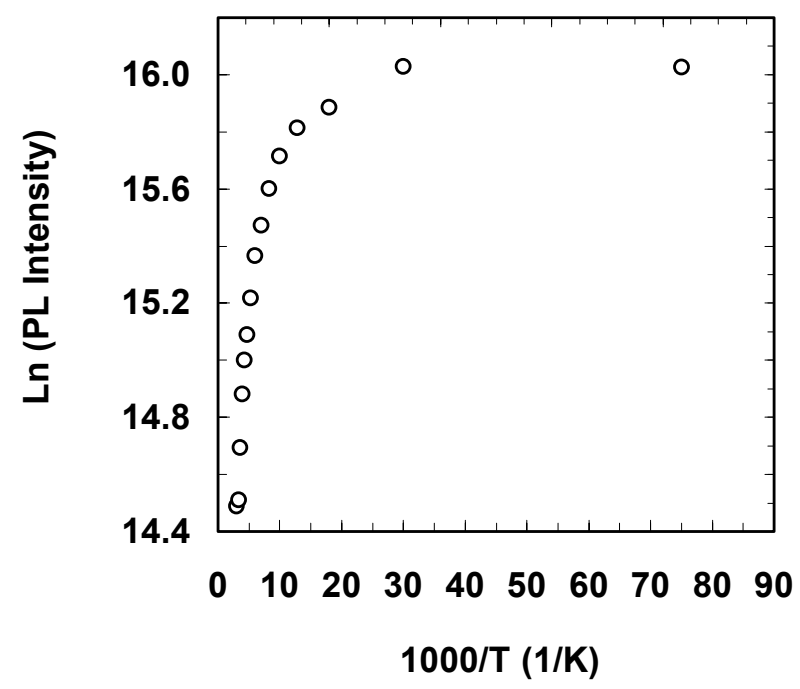

Figure 3.2. Temperature dependence of $\mathrm{a}-\mathrm{SiN}_{\mathrm{x}}: \mathrm{H} \mathrm{PL}$ intensity at the emission band maximum. Intensive quenching starts at $170 \mathrm{~K}$.

\subsection{Surface morphology of the amorphous silicon}

Atomic force microscopy (AFM) has been performed the on the a-SiN $\mathrm{N}_{\mathrm{x}}: \mathrm{H}$ samples to characterize the morphology of the surface and to analyze origin of the luminescence. The surface of the $\mathrm{a}-\mathrm{SiN}_{\mathrm{x}}: \mathrm{H}$ is optically flat and thus amenable for the growth of multiple layers. This is necessary condition for the realization of the one-dimensional PBG microcavity. The surface morphology of the a-SiNx:H is quite uniform. The bulk of the material is composed of globules of a-SiNx:H and is similar to п-Si. Figure 3.3 shows the medium resolution AFM picture of the silicon rich aSiNx:H surface. Additionally, although the surface is flat optically, it is quite rough in the $100 \mathrm{~nm}$ range, which corresponds to the average globule size. 


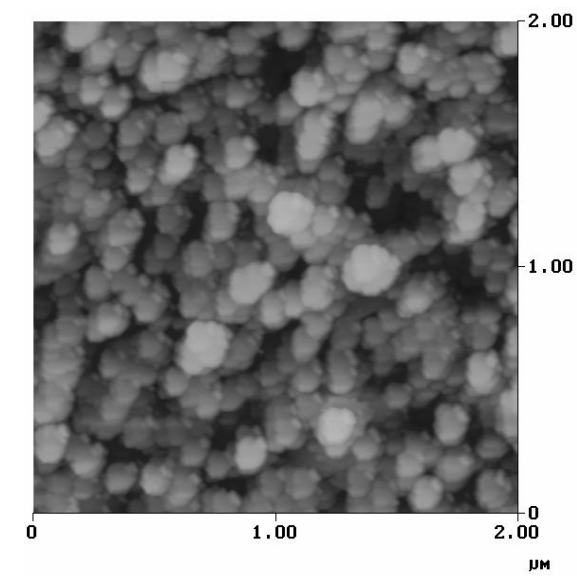

Figure 3.3. Medium resolution AFM of the silicon rich a-SiNx:H surface.

\section{POROUS SILICON}

Similar to hydrogenated amorphous silicon (a-Si:H), porous silicon (п-Si) also exhibits room temperature visible PL ${ }^{14}$. Semiconductor microcavity effects have been applied to $\pi-\mathrm{Si}$, after the observation of room temperature visible PL ) ${ }^{15}$ made $\pi$-Si a potential optical gain medium. ${ }^{16}$ Steady state ${ }^{17,18,19}$ and temporally resolved, ${ }^{20}$ single and multiple ${ }^{21}$ microcavity controlled PL in $\Pi-\mathrm{Si}$ has been observed experimentally ${ }^{22,23,24}$ and calculated theoretically ${ }^{25}$. The possibility of using $\Pi-\mathrm{Si}$ microcavities as chemical sensors has been investigated ${ }^{26}$. In addition, microcavity controlled $\mathrm{PL}$ has been observed in $\Pi$-Si inorganic-organic structures, ${ }^{27}$ as well as $\mathrm{Si} / \mathrm{SiO}_{\mathrm{x}}$ superlattices. ${ }^{28,29,30} \mathrm{SiO}_{2} / \mathrm{TiO}_{2}$ microcavities, ${ }^{31} \mathrm{SiO}_{\mathrm{x}} / \mathrm{WO}_{\mathrm{y}}$, and $\mathrm{SiO}_{\mathrm{x}} / \mathrm{MO}_{\mathrm{y}}$ multilayers ${ }^{32}$ have been fabricated. Microcavity controlled electroluminescence (EL) of $\Pi-\mathrm{Si}$ has been reported. ${ }^{33,}{ }^{34}$ Interference filters ${ }^{35}$ and optical waveguides ${ }^{36}$ have also formed from $\pi-\mathrm{Si}$. Two-dimensional (2-D) photonic crystals have been fabricated in $\pi-\mathrm{Si}^{37}$ and silicon nitride $\left(\mathrm{Si}_{3} \mathrm{~N}_{4}\right){ }^{38}$ waveguides.

\subsection{Temperature dependence of the porous silicon photoluminescence}

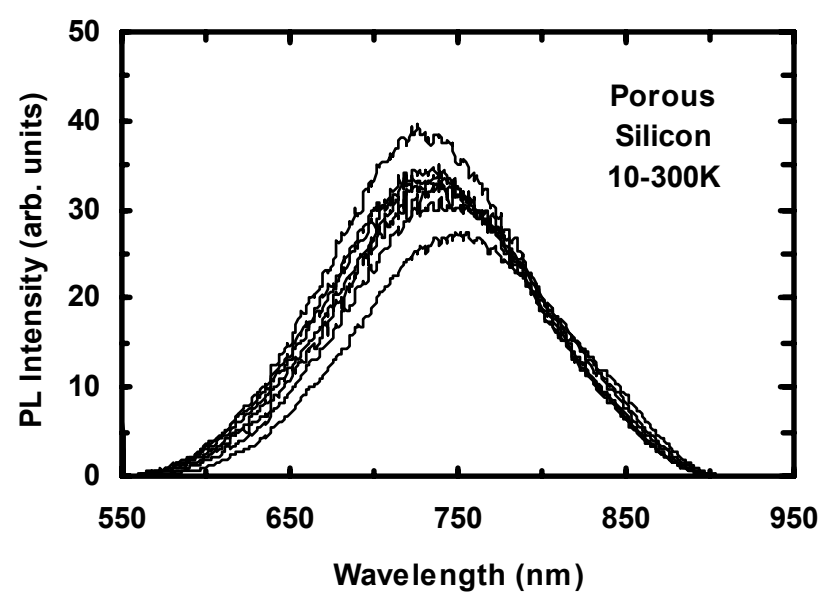

Figure 4.1. PL spectra of porous silicon in the 10 - $300 \mathrm{~K}$ temperature range. 


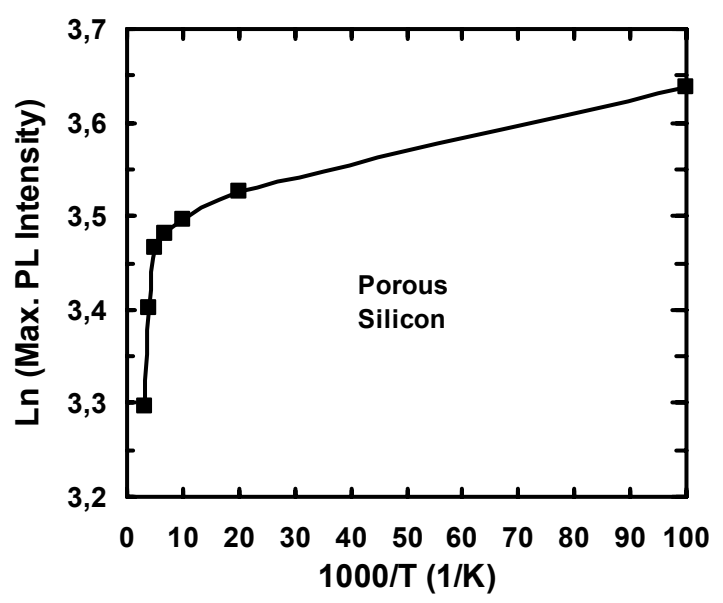

Figure 4.2. Temperature dependence of porous silicon PL intensity at the emission band maximum. Intensive quenching starts at $200 \mathrm{~K}$.

Figure 4.1 shows the PL spectra of the porous silicon measured in the $550-900 \mathrm{~nm}$ wavelength and in the $10-300 \mathrm{~K}$ temperature range at a constant excitation laser intensity of $0.005 \mathrm{~W} \mathrm{~cm}^{-2}$. A broad PL band centered at $750 \mathrm{~nm}\left(E_{p}=\right.$ $1.698 \mathrm{eV}$ ) at $10 \mathrm{~K}$ is observed. The PL spectra have approximately a Gaussian lineshape. The PL intensity decreases with increasing temperature. This feature is typical of the PL, which is due to donor-acceptor pair transitions observed in semiconductors. The variation of the PL peak with respect to temperature is plotted in the figure 4.2. In the 10 - 150 $\mathrm{K}$ range, the PL intensity decreases slowly. Above $200 \mathrm{~K}$, however, the PL intensity decreases at a larger rate due to a thermal quenching process. The activation energy $\Delta \mathrm{E}$ for this thermal quenching process can be derived in the $200-$ $300 \mathrm{~K}$ temperature range using a nonlinear least squares fit to the following equation, $\mathrm{I}=\mathrm{I}_{0} \exp \left(\Delta \mathrm{E} / \mathrm{k}_{\mathrm{B}} \mathrm{T}\right)$, where $\mathrm{I}$ is the PL intensity, $\mathrm{I}_{\mathrm{o}}$ a proportionality constant, and $\mathrm{k}_{\mathrm{B}}$ the Boltzmann's constant. The semilog plot of the emission band intensity as a function of the reciprocal temperature gives a straight line in the $200-300 \mathrm{~K}$ region. An activation energy of $E_{a}=0.009 \mathrm{eV}$ for the emission band is derived from the slope of the straight line fit. This activation energy is associated with a shallow level located at $0.009 \mathrm{eV}$ from the band. This shallow level in porous silicon may be associated with the presence of defects and unintentional impurities.

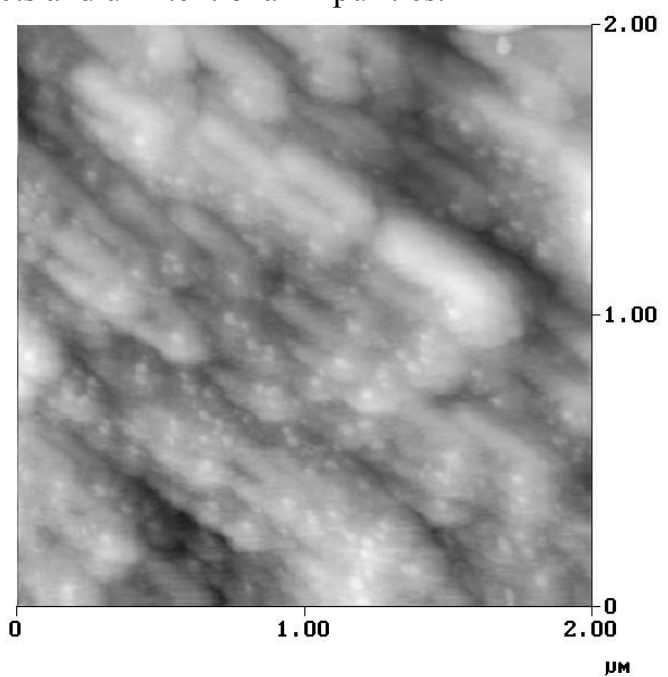

Figure 4.3. Medium resolution AFM of the porous silicon surface. 


\subsection{Surface morphology of the porous silicon}

Atomic force microscopy (AFM) has been performed the on the porous silicon samples to characterize the morphology of the surface and to analyze origin of the luminescence. The surface of the porous silicon is rather rough. The bulk of the material is composed of globules of as we have seen in porous silicon. Figure 4.3 shows the medium resolution AFM picture of the porous silicon surface.

\section{BLACK SILICON}

Ordinarily, silicon absorbs a moderate amount of visible light, but a substantial amount of visible light is reflected as well, and infrared and ultraviolet light are transmitted through silicon or reflected from it with very little absorption. Spiked silicon surfaces, in contrast, absorb nearly all light at wavelengths ranging from the ultraviolet to the infrared. This suggests it may be very useful in improving the performance of existing silicon devices, such as photodetectors and photovoltaics. Mazur group recently discovered that irradiation of silicon surfaces with femtosecond laser pulses in the presence of a halogen containing gas transforms the flat, mirror-like surface of a silicon wafer into a forest of microscopic spikes. ${ }^{39,40}$ The spiked surface is strongly light-absorbing: the surface of silicon, normally gray and shiny, turns deep black; hence the name black silicon.

\subsection{Temperature dependence of the PL from the black silicon}

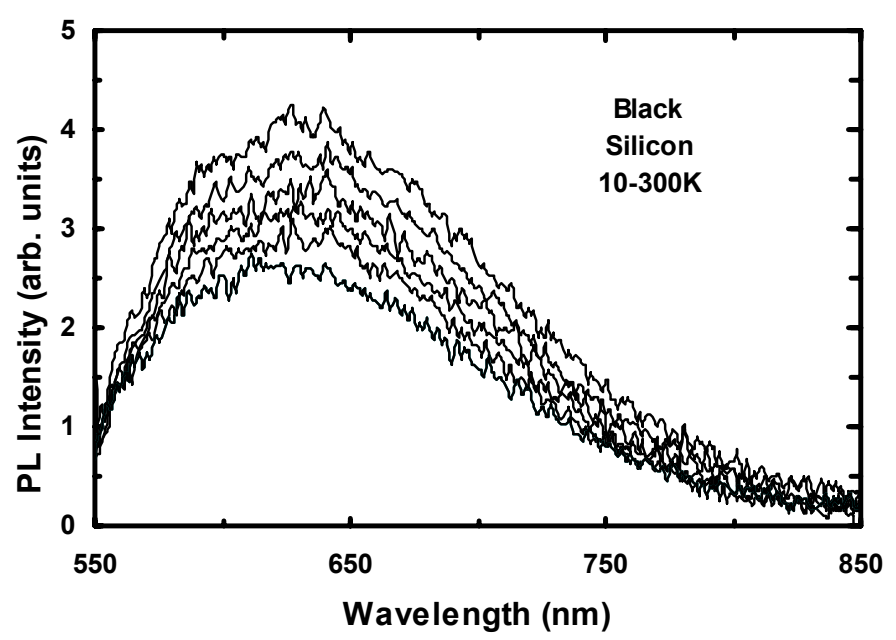

Figure 5.1. PL spectra of black silicon in the 10 - $300 \mathrm{~K}$ temperature range.

Figure 5.1 shows the PL spectra of the black silicon measured in the $550-850 \mathrm{~nm}$ wavelength and in the $10-300 \mathrm{~K}$ temperature range at a constant excitation laser intensity of $0.1 \mathrm{~W} \mathrm{~cm}^{-2}$. A broad PL band centered at $630 \mathrm{~nm}\left(E_{p}=\right.$ $1.968 \mathrm{eV}$ ) at $10 \mathrm{~K}$ is observed. The PL spectra have approximately Gaussian lineshape. The PL intensity decreases with increasing temperature. The variation of the PL peak with respect to temperature is plotted in the figure 2 . In the 10 $120 \mathrm{~K}$ range, the PL intensity decreases slowly. Above $120 \mathrm{~K}$, however, the PL intensity decreases at a larger rate due to a thermal quenching process. The activation energy $\triangle \mathrm{E}$ for this thermal quenching process can be derived in the 120 $300 \mathrm{~K}$ temperature range using a nonlinear least squares fit to the following equation, $\mathrm{I}=\mathrm{I}_{\mathrm{o}} \exp \left(\Delta \mathrm{E} / \mathrm{k}_{\mathrm{B}} \mathrm{T}\right)$, where $\mathrm{I}$ is the PL intensity, $\mathrm{I}_{\mathrm{o}}$ a proportionality constant, and $\mathrm{k}_{\mathrm{B}}$ the Boltzmann's constant. The semilog plot of the emission band intensity as a function of the reciprocal temperature gives a straight line in the $120-300 \mathrm{~K}$ region. An activation energy of $\mathrm{E}_{\mathrm{a}}=0.006 \mathrm{eV}$ for the emission band is derived from the slope of the straight line fit. This activation energy is associated with a shallow level located at $0.006 \mathrm{eV}$ from the band. This shallow level in black silicon may be associated with the presence of defects and unintentional impurities. 


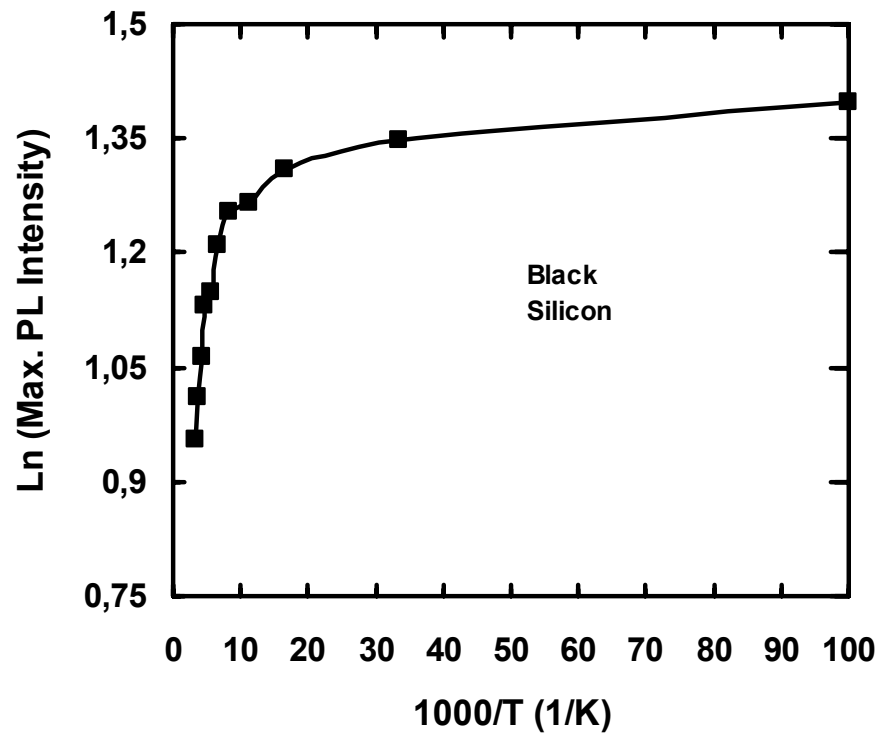

Figure 5.2. Temperature dependence of black silicon PL intensity at the emission band maximum. Intensive quenching starts at $120 \mathrm{~K}$.

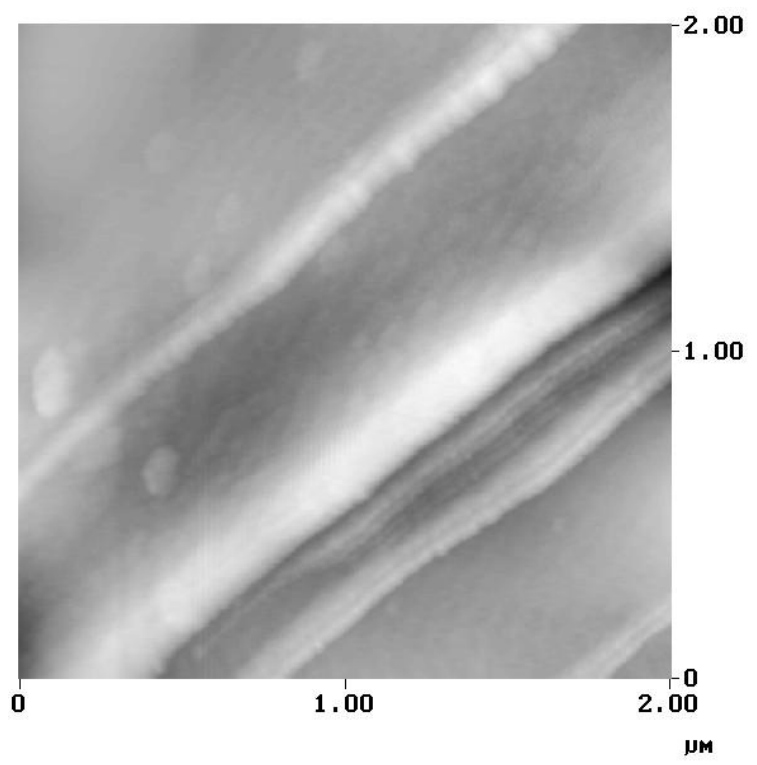

Figure 5.3. Medium resolution AFM of the black silicon surface. 


\subsection{Surface morphology of the black silicon}

Atomic force microscopy (AFM) has been performed the on the black silicon samples to characterize the morphology of the surface and to analyze origin of the luminescence. The surface of the black silicon is optically black and consists of conical layers. The bulk of the material is composed of globules of black silicon and is similar to п-Si. Figure 5.3 shows the medium resolution AFM picture of the black silicon surface.

\section{CONCLUSIONS}

Bulk luminescence properties of hydrogenated amorphous silicon nitride $\left(\mathrm{a}-\mathrm{SiN}_{\mathrm{x}}: \mathrm{H}\right)$, porous silicon, and black silicon were studied at room temperature and at low temperatures. The PL emission intensity increases at low temperatures due to the lack of thermally excited phonons. The emission spectrum stays broad even at low temperatures. The broad luminescence spectrum even at low temperatures is a results of the heterogenous size distribution of the amorphous silicon quantum dots. This heterogenous size distribution is further confirmed by atomic force microscopy (AFM) measurements of the sample surface. The broad PL spectrum of the a-SiN $\mathrm{x}: \mathrm{H}$, porous, and black silicon makes them suitable sources for wavelength division multiplexing (WDM) applications.

\section{ACKNOWLEDGMENTS}

We would like to acknowledge the partial support of this research by the Scientific and Technical Research Council of Turkey (TUBITAK) Grant No: TBAG-1952 and the European Office of Aerospace Research and Development (EOARD) Grants No: F61775-01-WE062 and FA8655-02-M-4086.

\section{REFERENCES}

1 H. Zimmermann, "Integrated Silicon Optoelectronics," (Springer Verlag, Berlin, 2000) pp. 1-352.

2 B. Jalali, S. Yegnanarayanan, T. Yoon, T. Yoshimoto, I. Rendina, and F. Coppinger, "Advances in Silicon on insulator Optoelectronics," IEEE J. Select. Topics in Quantum Electron. 4, 938 (1998).

3 A. Kaneko, T. Goh, H. Yamada, T. Tanaka, and I. Ogawa "Design and applications of silica-based planar lightwave circuits," IEEE J. Select. Topics in Quantum Electron. 5, 1227 (1999).

4 B. J. Offrein, R. Germann, F. Horst, H.W.M. Salemink, R. Beyerl, and G. L. Bona, "Resonant coupler-based tunable add-afterdrop filter in silicon-oxynitride technology for WDM networks," IEEE J. Select. Topics in Quantum Electron. 5, 1400 (1999).

5 J.D. Joannopoulos and G. Lucovsky, "The physics of hydrogenated amorphous silicon," (Springer Verlag, Berlin, 1984).

6 S. Perkowitz, "Optical Characterization of Semiconductors," (Academic Press, London, 1993), p.27.

7 P. Shaoqi and D. Xiaoning, "Properties of Sputtered Amorphous Silicon without Hydrogen", in "Proceedings of the International Workshop on Amorphous Semiconductors, "H. Fritzsche, D. Han, C. C. Tsai, Eds. (World Scientific, Singapore, 1987).

8 J. I. Panvoke, "Luminescence in Hydrogenated Amorphous Silicon", in "Proceedings of the International Workshop on Amorphous Semiconductors," H. Fritzsche, D. Han, C. C. Tsai, Eds., (World Scientific, Singapore, 1987).

9 R. A. Street, Ed., "Technology and Applications of Amorphous Silicon,” (Springer Verlag, Berlin, 2000), pp. 1-403.

10 A. M. Agarwal, L. Liao, J. S. Foresi, M. R. Black, X. Duan, and L.C. Kimerling, "Low-loss Polycrystalline Silicon Waveguides for Silicon Photonics," J. Appl. Phys. 80, 6120 (1996).

11 A. Serpengüzel, A. Aydinli, and A. Bek, "Enhancement and inhibition of photoluminescence in hydrogenated amorphous silicon nitride microcavities," Optics Express 1, 108 (1997).

12 M. H. Brodsky, "Quantum Well Model of the Hydrogenated Amorphous Silicon," Solid State Commun. 36, 55 - 59 (1980).

13 A. Serpengüzel, A. Aydinli, A. Bek, and M. Güre, "Visible photoluminescence from planar amorphous silicon nitride microcavities, " J. Opt. Soc. Am. B. 15, 2706 (1998).

14 D. J. Wolford, B. A. Scoot, J. A. Reimer and J. A. Bradley, "Efficient visible luminescence from hydrogenated amorphous silicon," Physica B 117, 920 (1983).

15 T. Canham, "Silicon Quantum Wire Array Fabrication by Electrochemical and Chemical Dissolution of Wafers," Appl. Phys. Lett. 57, 1046 (1990).

16 D. J. Lockwood, "Optical Properties of Porous Silicon," Solid State Commun. 92, 101 (1994).

17 L. Pavesi, C. Mazolleni, A. Tredicucci, and V. Pellegrini, "Controlled photon emission in porous silicon microcavities," Appl. Phys. Lett. 67, 3280 (1995). 
18 L. Pavesi and V. Mulloni, “All porous silicon microcavities: growth and physics,” J. Lumin. 80, 43 (1999).

19 M. Araki, H. Koyama, and N. Koshida, "Precisely tuned emission from porous silicon vertical optical cavity in the visible region," J. Appl. Phys. 80, 4841 (1996).

20 M. Cazzanelli and L. Pavesi, "Time-resolved photoluminescence of all porous silicon microcavities," Phys. Rev. B 56, 15264 (1997).

21 E. K. Squire, P. A. Snow, P. St. J. Russell, L. T. Canham, A. J. Simmons, and C. L. Reeves, "Light emission from porous silicon single and multiple cavities," J. Lumin. 80, 125 (1999).

22 M. Takahashi, Y. Toriumi, T. Matsumoto, Y. Masumoto, N. Koshida, "Significant photorefractive index change observed in porous silicon Fabry-Perot resonators,” Appl. Phys. Lett. 76, 1990 (2000).

23 S. Setzu, S. Letant, P. Solsona, R. Romestain, and J. C. Vial, " Improvement of luminescence in p-type as-prepared or dye impregnated porous silicon microcavities,” J. Lumin. 80, 129 (1999).

24 Z. H. Xiong, S. Yuan, Z. M. Jiang, J. Qin, C. W. Pei L. S. Liao, X. M. Ding, X. Y. Hou, and X. Wang, "Photoluminescence studies of porous silicon microcavities,” J. Lumin. 80, 137 (1999).

25 E. K. Squire, P. St. J. Russell, and P. A. Snow, "Optimized light emission from layered porous silicon structures,” Appl. Opt. 37, 7107 (1998).

26 V. Mulloni and L. Pavesi, "Porous silicon microcavities as optical chemical sensors," Appl. Phys. Lett. 76, 2523 (1999).

27 A. Arena, S. Patane, G. Saitta, S. Savasta, R. Girlanda, and R. Rinaldi, "Silicon based organic-inorganic microcavity and its dispersion curve from angle resolved photoluminescence," Appl. Phys. Lett. 72, 2571 (1998).

28 B. T. Sullivan, D. J. Lockwood, H. J. Labbe, and Z.-H. Lu, "Photoluminescence in Amorphous Si/SiOx Superlattices Fabricated by Magnetron Sputtering," Appl. Phys. Lett. 69, 3149 (1996).

29 D. J. Lockwood, J.M. Baribeau, and B. T. Sullivan, "Visible light from Si/SiOx superlattices in planar microcavities," J. Vac. Sci. Technol. B 16, 1707 (1998).

30 D. J. Lockwood, B. T. Sullivan, and H. J. Labbe, "Visible light emission from Si/SiOx superlattices in optical microcavities," J. Lumin 80, 75 (1999).

31 K. M. Cheng, A. W. Sparks, H.-C. Luan, D. R. Lim, K. Wada, and L. C. Kimerling, "SiO2/TiO2 omnidirectional reflector and resonator with sol-gel method," Appl. Phys. Lett. 75, 3805 (1999).

32 F. Hamelmann, G. Haindl, J. Schmalhorst, A. Aschentrup, E. Majkova, U. Kleineberg, U. Heinzmann, A. Klipp, P. Jutzi, A. Anopchenko, M. Jergel, and S. Luby, "Metal oxide / silicon oxide multilayer with smooth interfaces produced by in situ controlled plasma enhanced MOCVD," Thin Solid Films 358, 90 (2000).

33 L. Pavesi, R. Guardini, and C. Mazolleni, "Porous Silicon Resonant Cavity Light Emitting Diodes," Solid State Commun. 97, 1051 (1996).

34 M. Araki, H. Koyama, and N. Koshida, "Controlled electroluminescence spectra of porous silicon diodes with vertical optical cavity," Appl. Phys. Lett. 69, 2956 (1996).

35 D. Hunkel, R. Butz, R. Ares-Fisher, M. Marso, and H. Lüth, "Interference filters from porous silicon with laterally varying wavelength of reflection,” J. Lumin. 80, 133 (1999).

36 H. F. Arrand, T. M. Benson, P. Sewell, and A. Loni, "Optical waveguides in porous silicon pre-patterned by localized nitrogen implantation,” J. Lumin. 80, 199 (1999).

37 S. W. Leonard, H. M. van Driel, K. Busch, S. John, A. Birner, A.-P. Li, F. Müller, U Gösele, and V. Lehmann, "Attenuation of optical transmission within the band gap of thin two-dimensional macroporous silicon photonic crystals," Appl. Phys. Lett. 75, 3063 (1999).

38 M. C. Netti, M. D. B. Charlton, G. J. Parker, and J. J. Baumberg, "Visible photonic band gap engineering in silicon nitride waveguides," Appl. Phys. Lett. 76, 991 (2000).

39 Tsing-Hua Her, Richard J. Finlay, Claudia Wu, and Eric Mazur, "Femtosecond laser-induced formation of spikes on silicon", Appl. Phys. A 70, 383-385 (2000);

40 Tsing-Hua Her, Richard J. Finlay, Claudia Wu, Shrenik Deliwala, and Eric Mazur, "Microstructuring of silicon with femtosecond laser pulses", Appl. Phys. Lett. 73, 1673-1675 (1998). 